\title{
Focus on therapy: hemicrania continua and new daily persistent headache
}

\author{
Paolo Rossi $\cdot$ Cristina Tassorelli $\cdot$ Marta Allena $\cdot$ \\ Enrico Ferrante $\cdot$ Carlo Lisotto $\cdot$ Giuseppe Nappi
}

Received: 3 December 2009/ Accepted: 25 January 2010/Published online: 26 February 2010

(C) Springer-Verlag 2010

\begin{abstract}
Hemicrania continua (HC) and new daily-persistent headache (NDPH) represent the only two forms of chronic daily headache in Chap. IV "Other Primary Headaches" of the second edition of the International Classification of Headache Disorders. HC and NDPH are rare and poorly defined from a pathophysiological point of view; as a consequence, their management is largely empirical. Indeed, there is a lack of prospective, controlled trials in this field, and treatment effectiveness is basically inferred from the results of sparse open-label trials, retrospective case series, clinical experience and expert opinions. In this narrative review we have summarised the information collected from an extensive analysis of the literature on the treatment of HC and NDPH in order to provide the best available and up-to-date evidence for the management of these two rare forms of primary headache. Indomethacin is the mainstay of $\mathrm{HC}$ management. The reported effective
\end{abstract}

\author{
P. Rossi \\ Headache Clinic INI Grottaferrata, Rome, Italy \\ C. Tassorelli $(\bowtie) \cdot$ M. Allena $\cdot$ G. Nappi \\ Headache Science Centre, IRCCS “Neurological Institute C. \\ Mondino" Foundation, University Centre for Headache and \\ Adaptive Disorders (UCADH), University of Pavia, Pavia, Italy \\ e-mail: cristina.tassorelli@mondino.it \\ E. Ferrante \\ Department of Neuroscience, Niguarda Ca' Granda Hospital, \\ Milan, Italy \\ C. Lisotto \\ Headache Centre, Department of Neuroscience, \\ S. Vito al Tagliamento Hospital, \\ San Vito al Tagliamento (PN), Italy \\ G. Nappi \\ Chair of Neurology, University "La Sapienza", Rome, Italy
}

dose of indomethacin ranges from 50 to $300 \mathrm{mg} /$ day. Gabapentin 600-3,600 mg tid, topiramate $100 \mathrm{mg}$ bid, and celecoxib 200-400 mg represent the most interesting alternative choices in the patients who do not tolerate indomethacin or who have contraindications to its use. NDPH is very difficult to treat and it responds poorly only to first-line options used for migraine or tension-type headache.

Keywords Hemicrania continua $\cdot$ New daily persistent headache - Chronic daily headache · Therapy . Management

\section{Background}

Hemicrania continua (HC) and new daily-persistent headache (NDPH) represent the only two forms of chronic, daily headache in Chap. IV "Other Primary Headaches" of the second edition of the International Classification of Headache Disorders (ICHD-II) [1]. The chronic temporal pattern differentiates these two forms from the other types included in Chap. IV, which are episodic and/or shortlasting headache and rarely require a prolonged treatment.

Hemicrania continua (HC) and NDPH are rare and poorly defined from a pathophysiological point of view. As a consequence, the management of HC and NDPH is largely empirical. Indeed, there is a lack of prospective, controlled trials in this field, and treatment effectiveness is basically inferred from the results of sparse open-label trials, retrospective case series, clinical experience and expert opinions. The only available guidelines for the therapy of HC and NDPH are not available in English and have been released soon after the publication of ICHD-II, thus including cases mainly diagnosed with other diagnostic criteria [2]. 
In this narrative review we have summarised the information collected from an extensive analysis of the literature on the treatment of $\mathrm{HC}$ and NDPH in order to provide the best available and up-to-date evidence for the management of these two rare forms of primary headache.

\section{Hemicrania continua}

Clinical features, diagnostic criteria, epidemiology and pathophysiology

Hemicrania continua (HC) is an uncommon primary headache disorder, first described as a syndrome by Sjaastad and Spierings in 1984 [3]. Hemicrania continua is a strictly unilateral, continuous, moderate-to-severe headache that fluctuates in intensity with possible exacerbations of severe pain associated with autonomic disturbances (conjunctival injection and/or lacrimation, nasal congestion and/or rhinorrhoea, ptosis and/or miosis); it is absolutely responsive to indomethacin (Table 1, diagnostic criteria). Migrainous features and jab-and-jolt pain may also be present [4]. Hemicrania continua is usually unremitting, but rare cases of remission have been reported. It is probably less uncommon than thought in the past [4]. In the Vågå study of headache epidemiology [5], up to $1 \%$ of the individuals surveyed exhibited a clinical picture that seemed to resemble HC but the diagnosis could not be confirmed for the compliance problems of the study (difficult to assess the response to indomethacin).

Different classification systems have been proposed for HC [6, 7]. Universally accepted operational diagnostic criteria for HC are contained in the ICHD-II and include, as an obligatory criterion, an absolute response to therapeutic doses of indomethacin. However, this criterion has been criticised because $\mathrm{HC}$ may also respond to other drugs, although less effectively, and also because it means that HC cannot be diagnosed in patients who have never been administered this drug [7]. Very recently, Murmura et al. [8], in a retrospective study, reported that most patient with a clinical phenotype leading to a putative diagnosis of $\mathrm{HC}$ do not respond to indomethacin. The fact that $\mathrm{HC}$ is included in Chap. IV of ICHD-II, "Other primary headaches", reflects its uncertain nosological status. Indeed, the clinical phenotype of $\mathrm{HC}$ overlaps that of trigeminal autonomic cephalalgias and migraine, possibly because it shares a pathophysiological mechanism with these two conditions [9, 10]. The inclusion of $\mathrm{HC}$ among the primary chronic headache disorders (CHDs) has been criticised on the grounds that aside from the chronicity, a highly unspecific quality, $\mathrm{HC}$ is a headache condition showing a sharply delineated unilaterality and clear therapeutic profile [11]. From a practical point of view, it may indeed be helpful to consider $\mathrm{HC}$ as one of the possible causes of chronic daily headache (CDH). Early administration of the "indotest" (a diagnostic test to detect indomethacin-responsive headaches) to all cases of chronic unilateral headache may lead to the timely identification of cases of $\mathrm{HC}$ [4].

Early diagnosis of $\mathrm{HC}$ is mandatory because the condition can be highly disabling and treatment with indomethacin may help patients to achieve a pain-free state. The diagnostic indotest involves injection of indomethacin $50 \mathrm{mg}$ i.m. and measurement of time for complete pain relief. In 12 HC patients, complete pain relief was achieved $2 \mathrm{~h}$ after this injection [12]. The test is simple and may also be helpful in identifying atypical cases [13].

\section{Treatment}

Indomethacin is the mainstay of $\mathrm{HC}$ management. The mechanism by which it exerts its effect on $\mathrm{HC}$ and other primary headaches is unclear. The reported effective dose of indomethacin for $\mathrm{HC}$ ranges from 50 to $300 \mathrm{mg}$ a day [3, 14]. It is advisable to start with $25 \mathrm{mg}$ tid. The response to
Table 1 International Headache Society diagnostic criteria for hemicrania continua

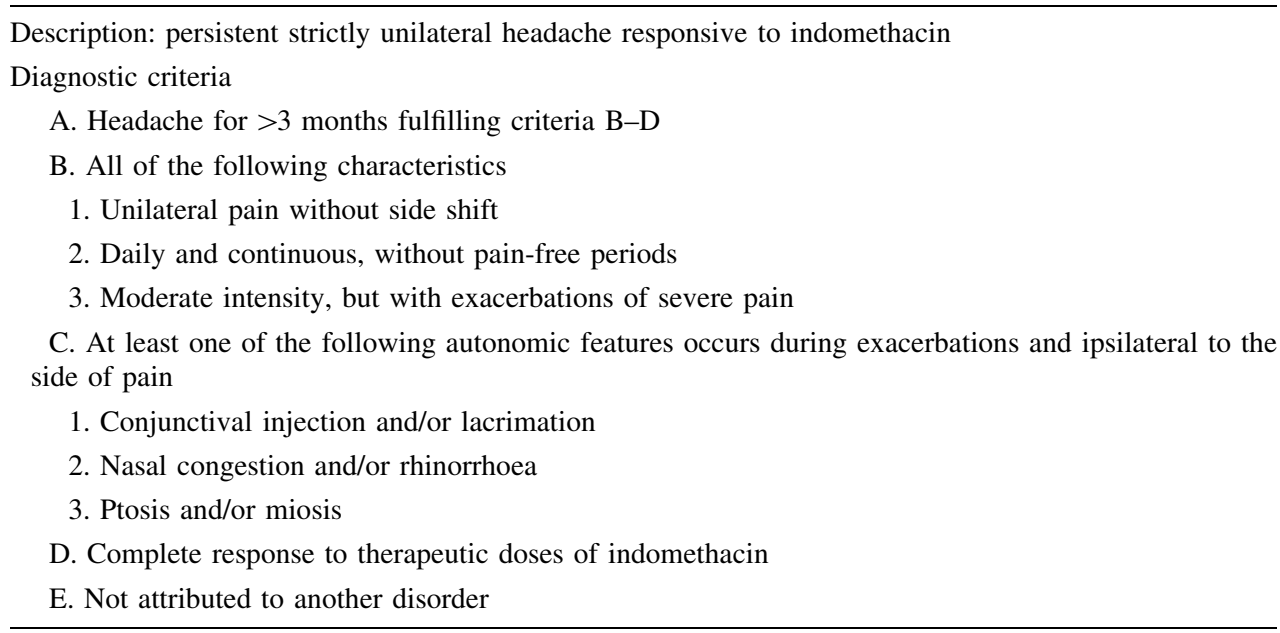


indomethacin is prompt. Most patients report complete relief of headache within $24 \mathrm{~h}$. After one week, if the patient is asymptomatic, the dose should be decreased to the minimum effective dose at which the patient remains pain-free. If, on the other hand, there is no satisfactory response, the dose should be increased to $50 \mathrm{mg}$ tid. Occasional patients require higher doses. Gastroprotectors are required to control gastrointestinal side effects of indomethacin and to prevent gastroduodenal ulcers. Because remitting forms have been described, after 3-6 months all patients should try to decrease the daily dose of indomethacin by $25 \mathrm{mg}$ each week until discontinuation, unless the symptoms reappear.

Hemicrania continua is often a lifelong condition, raising the issue of loss of therapeutic efficacy over time and potential long-term side effects. Indomethacin does not seem to show tachyphylaxis. On the contrary, Pareja et al. [6] showed that, over time, $42 \%$ of patients were able to decrease, by up to $60 \%$, the dose of indomethacin required to maintain a pain-free state. As regards the long-term tolerability of indomethacin, of $12 \mathrm{HC}$ patients followed up for varying periods of between 1 and 11 years, 23\% experienced minor side effects, mostly gastrointestinal problems relieved with gastroprotectors [4]. In the literature, more than $35 \%$ of patients receiving therapeutic dosages of indomethacin experience adverse effects and $20 \%$ have to discontinue the drug [15]. Most adverse effects are dose-related, which underlines the importance of achieving the lowest effective dose. Gastrointestinal complications, such as anorexia, nausea, vomiting, dyspepsia, abdominal pain, mucosal ulceration and diarrhoea are the most frequent adverse effects but they are not often a cause for discontinuation. Indomethacin is contraindicated in conditions, such as renal failure, gastric ulcers and bleeding disorders. The risks associated with long-term indomethacin use include gastrointestinal ulcers and renal dysfunction, such as papillary necrosis. The first described HC patient was followed up for 19 years and developed bleeding gastric ulcer, treated with gastric surgery [16].

Patients who cannot tolerate indomethacin present a major challenge as regards the management of their headache, as no other drug has been shown to be consistently effective in HC. However, anecdotal observations suggest that drugs other than indomethacin may be helpful in HC. Sjaastad and Antonaci [17] reported a complete response to piroxicam beta-cyclodestrin 20 to $40 \mathrm{mg} /$ day in 4 out of 6 patients ( 1 had a moderate response and 1 had no response). Selective COX-2 inhibitors, rofecoxib (50 mg/day) and celecoxib (200-400 mg bid), were found to be highly effective in 3 out of 9 patients and 3 out of 5 patients [18], respectively ( 1 patient receiving celecoxib and 5 of those receiving rofecoxib experienced a partial response). The COX-2 inhibitors have been proposed as an alternative to indomethacin in $\mathrm{HC}$ and other indomethacin-responsive syndromes [18, 19], but their long-term use has recently been associated with an increased risk of myocardial infarction and stroke, and rofecoxib has been withdrawn from the market worldwide [20]. Indeed, an increased risk of cardiovascular events exists with non-steroideal anti-inflammatory drugs in general, indomethacin included, and patients with cardiovascular disease should be informed about this risk and considered for alternative therapeutic options [21]. Rozen [22] described 3 patients responding to melatonin (9$15 \mathrm{mg} /$ day), which has a similar chemical structure to indomethacin. In two cases melatonin alone was sufficient to achieve a pain-free state while in the third case it made it possible to reduce the total dose of indomethacin by $50 \%$. Another case responding to melatonin $7 \mathrm{mg}$ at bedtime was described by Spears [23], and there have recently been descriptions of five cases (two with atypical features) responding to topiramate (100 $\mathrm{mg}$ bid), and of a single patient with $\mathrm{HC}$ evolving from $\mathrm{CH}$ responding to valproic acid [24-27]. Very recently, Spears reported the efficacy of gabapentin in $9 \mathrm{HC}$ patients who had difficulties in tolerating indomethacin [28]. Seven patients reported a 50-80\% reduction of pain with doses ranging from 900 to $3,600 \mathrm{mg} /$ day (two of them used other medication for pain control). One patient reported a $50 \%$ reduction of pain and one reported no effect. Four patients were pain free on gabapentin (600-1,800 mg/day).

Isolated case reports have described ibuprofen, naproxen, aspirin, paracetamol with caffeine and verapamil as effective [29, 30], but most of these drugs have been found to be ineffective in other $\mathrm{HC}$ cases.

Other classes of drug have not been successful in controlling HC. Antonaci et al. [31] reported a lack of efficacy of sumatriptan in 7 patients. Because $\mathrm{HC}$ is widely misdiagnosed, patients are prescribed many classes of drugs, often ones effective in migraine (such as analgesics, calcium-antagonists, beta-blockers, amitriptyline and other antidepressants, antiepileptics, ergot derivatives, pizotifen, methysergide) as well as others that are reported to be of no benefit in migraine [32].

Anaesthetic blockades of pericranial nerves have been found to be ineffective [33].

Very recently, Schwedt et al. and Burns et al. [34, 35] reported that occipital nerve stimulation may be a safe and effective treatment for HC (8 cases) at short- and long-term follow-up.

To review the problems linked to the misdiagnosis and mismanagement of $\mathrm{HC}$ patients we recently interviewed 25 consecutive $\mathrm{HC}$ subjects attending the Headache Clinic INI Grottaferrata [36]. Patients were asked about their use of pharmacological treatments, surgical treatments and nonpharmacological treatments for headache and to rate the effectiveness of each treatment on a four-point scale (very effective, i.e. complete and long-lasting relief; effective, 
i.e. partial and/or short-lasting relief; ineffective; headache worsened). The patients had tried a mean of $3.63 \pm 2.1$ different classes of drug $(67 \%$ prescribed, $33 \%$ unprescribed). NSAIDs had been tried by $92 \%$. Nimesulide (a non-steroideal anti-inflammatory drug not available on the market in US and in the majority of EU countries) had been tried by 7 patients and been judged very effective by one and effective by six. Aspirin and ibuprofen had each been tried by 9 patients and were rated as effective by five and three of them, respectively. Antidepressants had been used by 8 patients ( 6 amitriptyline, 2 fluoxetine) but showed no effectiveness. Triptans had been used by 8 patients $(5$ sumatriptan s.c., 1 zolmitriptan and 2 rizatriptan) and been judged ineffective by all of them. Two patients had used rofecoxib and considered it very effective. Taken together, these HC patients had used a cumulative total of 80 different drug treatments, judging $73.7 \%$ of these medications ineffective, $22.5 \%$ partially effective (all NSAIDs) and $3.75 \%$ (rofecoxib and nimesulide) effective.

As much as $36 \%$ of the patients had undergone ineffective and unnecessary surgery (dental extraction, sinus/ deviated septum surgery, temporomandibular joint surgery and cervical spine surgery) for their HC. Four patients had tried acupuncture (two considering it effective) and four had tried homeopathy (deemed ineffective by all of them).

These data suggest that $\mathrm{HC}$ is largely mismanaged as a consequence of its misdiagnosis. Indeed, apart from NSAIDs which, as a rule, were not prescribed, patients were mainly prescribed, by physicians, medications shown to be effective in the treatment of migraine or cluster headache but ineffective for $\mathrm{HC}$.

Hemicrania continua may be complicated by overuse of symptomatic drugs [8, 37] and (in this situation) differential diagnosis of $\mathrm{HC}$ versus transformed or chronic migraine may be difficult. An exhaustive disease history could be helpful, as it may show a pre-existing unilateral primary headache. However, the overused medication should always be withdrawn and, if the headache persists, the indomethacin response should be tested.

Figure 1 sets out a schematic approach to the management of $\mathrm{HC}$.
Fig. 1 A schematic approach to the management of hemicrania continua

\section{First choice}

Indomethacin 50-300 mg

- Start with $25 \mathrm{mg}$ tid with me als and increase the dosage gradually until complete pain relief is obtained

- $\quad$ Treatment failure should be considered if a patient fails to respond to a daily dosage of 300 mg (consider alternative diagnosis)

- Once an effective dosage has been established for several weeks, reduce the dosage to ascertain the lowest effective dosage

- $\quad$ Prescribe gastroprotectors to prevent and manage gastrointestinal side effects

- $\quad$ Check renal function regularly

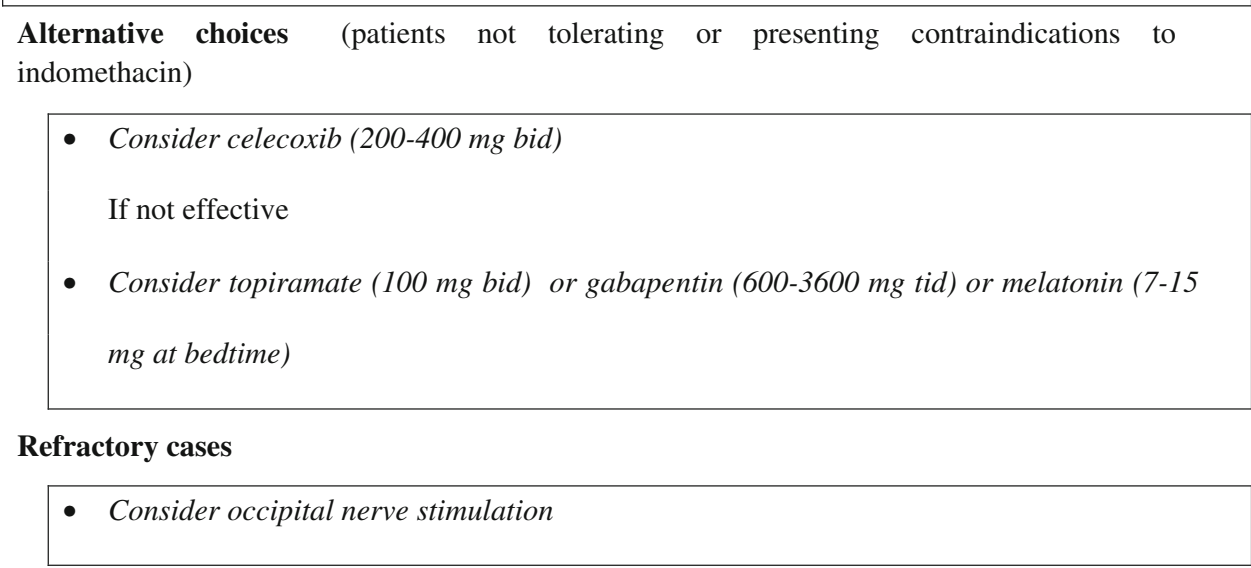




\section{New daily-persistent headache (NDPH)}

Clinical features, diagnostic criteria, epidemiology and pathophysiology

New daily-persistent headache (NDPH) is characterised by the abrupt onset of persistent headache that generally develops over $<3$ days and does not remit (Table 2, diagnostic criteria). In isolated reports on this entity, NDPH has been interpreted as a post-viral syndrome [38] and described as having a spontaneously favourable outcome [39]. On the basis of retrospective clinical observations, Silberstein et al. [40] included NDPH as a separate clinical entity in their classification of $\mathrm{CDH}$ and provided operational diagnostic criteria for the condition [briefly, they included: (a) average headache frequency $>15$ days/month for $>1$ month, (b) average headache duration $>4 \mathrm{~h} /$ day, if untreated, (c) no history of migraine or TTH increasing in frequency or decreasing in severity in association with the onset of NDPH, (d) acute onset-developing over 3 daysof constant unremitting headache and exclusion of secondary headache]. In ICHD-II, NDPH is included in Chap. IV, "Other primary headaches", underlining its uncertain nosological status. According to these International Headache Society diagnostic criteria, NDPH is phenotypically reminiscent of tension-type headache (TTH), i.e. a sort of de novo chronic TTH (CTTH). However, NDPH is unique in that the headache is daily and unremitting from or almost from the moment of onset, and occurs typically in individuals without a prior headache history, which suggests that the pathogenetic mechanisms in NDPH and CTTH are different.

In a recent clinic-based study conducted in a paediatric population, using a modified version of the ICHD-II criteria, NDPH was more common than CTTH and most of the subjects with NDPH did not overuse medication and commonly presented migrainous features [41].

New daily-persistent headache (NDPH) is probably a heterogeneous disorder and should therefore be regarded as a syndrome. A viral infection or other organic cause may precede the headache in more than one-third of patients [42], possibly leading to a CNS inflammation and sensitisation of nociceptive pathways [43]. In some patients, cervical spine joint hypermobility may be a factor predisposing to the development of NDPH [44]. NDPH has a wide range of secondary forms that have to be excluded after thorough diagnostic work-up [45]. In these cases the causes (e.g. spontaneous intracranial hypotension, neoplasms, pseudotumour cerebri, cervical artery dissections, cerebral venous thrombosis, Chiari I malformation and temporal arteritis) can be treated and should be carefully excluded before a headache management plan is worked out [45]. The prognosis of NDPH is highly variable, ranging from self-limiting cases that typically resolve without therapy within several months to refractory cases resistant to aggressive treatment programmes.

Further pathophysiological and clinical characterisation of this syndrome is necessary so that the management of NDPH can be based on a clear rationale and on specific treatment options and general recommendation can be given. Empirical evidence on NDPH therapy is poor and based on the application of treatments that have proved to be effective in migraine or TTH. No prospective, placebocontrolled trial has been conducted in this field and the effectiveness of treatment can only be inferred, from the results of a few open-label trials, retrospective case reviews, anecdotal observations, expert opinions and generalisations from the literature on episodic migraine and TTH.
Table 2 International Headache Society diagnostic criteria for NDPH

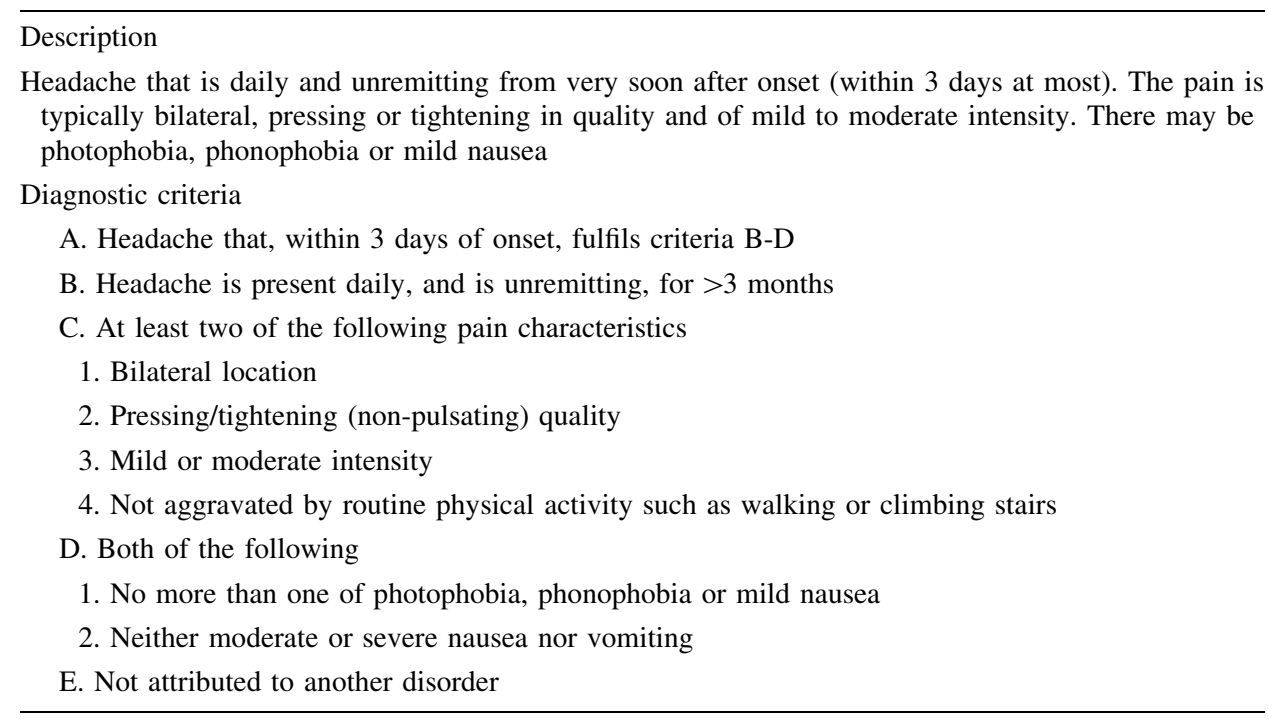


Treatment

The largest uncontrolled study investigating the effectiveness of drug therapy and the prognosis of NDPH diagnosed according to the ICHD-II criteria was conducted in Japan by Takase et al. [46]. In $30 \mathrm{NDPH}$ patients (17 males) the authors first administered muscle relaxants. If no effect was observed, tricyclic antidepressants (23 patients), valproic acid (9 patients), SSRIs (12 patients) and beta-blockers (2 patients) were subsequently administered. Drug treatment was rated as very effective by $27 \%$ of patients, moderately effective by $3 \%$, mildly effective by $20 \%$ and ineffective by $50 \%$. The authors concluded that NDPH has a poor prognosis and is highly resistant to currently available treatments.

Meineri et al. [47] retrospectively evaluated the effectiveness of drug therapy in $18 \mathrm{NDPH}$ patients (the authors used both ICHD-II and Silberstein-Lipton's criteria). Sixteen patients tried amitriptyline, seven tried fluoxetine and seven tried valproic acid. The authors reported that no drug was effective.

In a small American case series of NDPH patients diagnosed according to the Silberstein and Lipton criteria, the following drugs were reported to be effective: gabapentin ( 1 case, $2,700 \mathrm{mg} /$ day), topiramate ( 1 case, $150 \mathrm{mg}$ / day), venlafaxine (1 case, $75 \mathrm{mg} /$ day) and nortriptyline (1 case, $100 \mathrm{mg} /$ day) [48]. In these cases the therapeutic effectiveness was achieved after the patients had tried many first-line drugs for migraine and CTTH. Marmura et al. [49] recently reported 3 patients with NDPH (two of which were overusing symptomatic drugs) who experienced significant improvement with mexiletine (1,050$1,200 \mathrm{mg}$ ) after having failed on multiple appropriate preventive treatments. All of these patients experienced side effects, such as nausea, fatigue, tremor and dizziness, which were reported to be dose-dependent. An isolated report has documented the effectiveness of botulinum toxin A [50].

Like other chronic daily headaches, NDPH may be complicated by medication overuse. Physicians are advised to ascertain a patient's complete medication history before starting any therapy. If medication overuse is diagnosed, drug withdrawal is necessary before other therapeutic options can be tried even though no prospective study has specifically investigated the effect of medication overuse in the worsening and maintenance of NDPH or in the determining of a resistance to therapy.

In summary, NDPH seems to be difficult to treat and to respond only poorly to first-line options used for migraine or TTH. Well-designed, targeted clinical trials considering the heterogeneity of this clinical entity, are needed so that an evidence-based therapy can be developed for this poorly characterised clinical syndrome.
Conflict of interest None.

\section{References}

1. International Headache Society Classification Subcommittee (2004) International classification of headache disorders, 2nd edn. Cephalalgia 24 (Suppl 1):1-60

2. Evers S, Frese A, May A, Sixt G, Straube A. Die therapie seltener idiopatischer Kopfschmerzerkrankungen - Empfehlungen der Deutschen Migrane und Kopfschmerzgesellschaft. http://www. dmkg.de/pdf/selt_idiop_ks.pdf. Accessed 12 Jan 2010

3. Sjaastad O, Spierings EL (1984) "Hemicrania continua": another headache absolutely responsive to indomethacin. Cephalalgia 4:65-70

4. Peres MF, Silberstein SD, Nahmias S, Schechter AL, Youssef I, Rozen TD, Young WB (2001) Hemicrania continua is not that rare. Neurology 57:948-951

5. Sjaastad O, Bakketeig LS (2007) The rare, unilateral headaches. Vågå study of headache epidemiology. J Headache Pain 8:19-27

6. Pareja JA, Vincent M, Antonaci F, Sjaastad O (2001) Hemicrania continua: diagnostic criteria and nosologic status. Cephalalgia 21:874-877

7. Goadsby PJ, Lipton RB (1997) A review of paroxysmal hemicranias, SUNCT syndrome and other short-lasting headaches with autonomic features, including new cases. Brain 120:193-209

8. Murmura MJ, Silberstein SD, Gupta M (2008) Hemicrania continua: who responds to indomethacin? Cephalalgia 29:300-307

9. Allena M, Tassorelli C, Sances G, Guaschino E, Sandrini G, Nappi G, Antonaci F (2008) Is hemicrania continua a single entity or the association of two headache forms? Considerations from a case report. Headache Oct 10 (Epub ahead of print)

10. Matharu MS, Cohen AS, McGonigle DJ, Ward N, Frackowiak RS, Goadsby PJ (2004) Posterior hypothalamic and brainstem activation in hemicrania continua. Headache 44:747-761

11. Spierings EL, Sjaastad O (2002) Hemicrania continua is not that rare. Neurology 59:476-477

12. Antonaci F, Pareja JA, Caminero AB, Sjaastad O (1998) Chronic paroxysmal hemicrania and hemicrania continua. Parenteral indomethacin: the "indotest". Headache 38:122-128

13. Baldacci F, Nuti A, Cafforio G, Lucetti C, Logi C, Cipriani G, Orlandi G, Bonuccelli U (2008) 'INDOTEST' in atypical hemicrania continua. Cephalalgia 28:300-301

14. Pareja JA, Caminero AB, Franco E, Casado JL, Pascual J, Sánchez del Río M (2001) Dose, efficacy and tolerability of longterm indomethacin treatment of chronic paroxysmal hemicrania and hemicrania continua. Cephalalgia 21:906-910

15. Dodick D (2004) Indomethacin-responsive headache syndromes. Curr Pain Headache Rep 8:19-26

16. Sjaastad O (2006) Chronic paroxysmal hemicrania, hemicrania continua and SUNCT: the fate of the three-first described cases. J Headache Pain 7:151-156

17. Sjaastad O, Antonaci F (1995) A piroxicam derivative partly effective in chronic paroxysmal hemicrania and hemicrania continua. Headache 35:549-550

18. Peres MF, Silberstein SD (2002) Hemicrania continua responds to cyclooxygenase-2 inhibitors. Headache 42:530-531

19. Peres MF, Zukerman E (2000) Hemicrania continua responsive to rofecoxib. Cephalalgia 20:130-131

20. Lenzer J (2005) FDA advisers warn: COX 2 inhibitors increase risk of heart attack and stroke. BMJ 330:440

21. Roumie CL, Choma NN, Kaltenbacch L, Mitchel EF Jr, Arbogast PG, Mr Griffin (2009) Non-aspirin NSAIDs, cyclooxygenase-2 inhibitors and risk for cardiovascular events-stroke, acute 
yocardial infarction, and death from coronary hearth disease. Pharmacoepidemiol Drug Saf 18:1053-1063

22. Rozen TD (2006) Melatonin responsive hemicrania continua. Headache 46:1203-1204

23. Spears RC (2006) Hemicrania continua: a case in which a patient experienced complete relief on melatonin. Headache 46:524-527

24. Matharu MS, Bradbury P, Swash M (2006) Hemicrania continua: side alternation and response to topiramate. Cephalalgia 26:341344

25. Brighina F, Palermo A, Cosentino G, Fierro B (2007) Prophylaxis of hemicrania continua: two new cases effectively treated with topiramate. Headache 47:441-443

26. Camarda C, Camarda R, Monastero R (2008) Chronic paroxysmal hemicrania and hemicrania continua responding to topiramate: two case reports. Clin Neurol Neurosurg 110:88-91

27. Lambru G, Castellini P, Bini A, Evangelista A, Manzoni GC, Torelli P (2008) Hemicrania continua evolving from cluster headache responsive to valproic acid. Headache 48:1374-1376

28. Spears RC (2009) Is gabapentin an effective treatment choice for hemicrania continua? J Headache Pain 10:271-275

29. Matharu MS, Boes CJ, Goadsby PJ (2003) Management of trigeminal autonomic cephalalgias and hemicrania continua. Drugs 63:1637-1677

30. Rajabally YA, Jacob S (2005) Hemicrania continua responsive to verapamil. Headache 45:1082-1083

31. Antonaci F, Pareja JA, Caminero AB, Sjaastad O (1998) Chronic paroxysmal hemicrania and hemicrania continua: lack of efficacy of sumatriptan. Headache 38:197-200

32. Bordini C, Antonaci F, Stovner LJ, Schrader H, Sjaastad O (1991) "Hemicrania continua": a clinical review. Headache 31:20-26

33. Antonaci F, Pareja JA, Caminero AB, Sjaastad O (1997) Chronic paroxysmal hemicrania and hemicrania continua: anaesthetic blockades of pericranial nerves. Funct Neurol 12:11-15

34. Schwedt TJ, Dodick DW, Hentz J, Trentman TL, Zimmerman RS (2007) Occipital nerve stimulation for chronic headache-long term safety and efficacy. Cephalalgia 27:153-157

35. Burns B, Watkins L, Goadsby PJ (2008) Treatment of hemicrania continua by occipital nerve stimulation with a bion device: longterm follow-up of a crossover study. Lancet Neurol 7:1001-1012
36. Rossi P, Faroni V, Tassorelli C, Nappi G (2009) Diagnostic delay and mismanagement in a referral population with hemicrania continua. Headache 49:227-234

37. Pareja JA, Antonaci F, Vincent M (2001) The hemicrania continua diagnosis. Cephalalgia 21:940-946

38. Diaz-Mitoma F, Vanast WJ, Tyrrel DL (1987) Increased frequency of Epstein-Barr virus excretion in patients with new-daily persistent headaches. Lancet 1:411-415

39. Vanast WJ (1986) New daily persistent headache: definition of a benign syndrome. Headache 26:317

40. Silberstein SD, Lipton RB, Solomon S, Mathew NT (1994) Classification of daily and near daily headache: proposed revision to the IHS-criteria. Headache 34:1-7

41. Kung E, Tepper SJ, Rapoport AM, Sheftell FD, Bigal ME (2009) New daily persistent headache in the paediatric population. Cephalalgia 29:17-22

42. Li D, Rozen TD (2002) The clinical characteristics of new daily persistent headache. Cephalalgia 22:66-69

43. Rozen T, Swidan SZ (2007) Elevation of CSF tumor necrosis factor alpha levels in new daily-persistent headache and treatment refractory chronic migraine. Headache 47:1050-1055

44. Rozen TD, Roth JM, Denenberg N (2006) Cervical spine joint hypermobility: a possible predisposing factor for new daily persistent headache. Cephalalgia 26:1182-1185

45. Goadsby PJ, Boes C (2002) New daily persistent headache. J Neurol Neurosurg Psychiatry 72(Suppl 2):ii6-ii9

46. Takase Y, Nakano M, Tatsumi C, Matsuyama T (2004) Clinical features, effectiveness of drug-based treatment, and prognosis of new daily persistent headache (NDPH): 30 cases in Japan. Cephalalgia 24:955-959

47. Meineri P, Torre E, Rota E, Grasso E (2004) New daily persistent headache: clinical and serological characteristics in a retrospective study. Neurol Sci 25(Suppl 3):S281-S282

48. Evans RW, Rozen TD (2001) Etiology and treatment of new daily persistent headache. Headache 41:830-832

49. Marmura MJ, Passero FC Jr, Young WB (2008) Mexiletine for refractory chronic headache: a report of nine cases. Headache 48:1506-1510

50. Spears RC (2008) Efficacy of botulinum toxin type A in new daily persistent headache. J Headache Pain 9:405-406 